\title{
Solid-State Characterization and Interconversion of Recrystallized Amodiaquine Dihydrochloride in Aliphatic Monohydric Alcohols
}

\author{
Wiriyaporn Sirikun, ${ }^{1}$ Jittima Chatchawalsaisin, ${ }^{1,2}$ and Narueporn Sutanthavibul ${ }^{1,2,3}$
}

Received 28 April 2015; accepted 11 June 2015; published online 24 July 2015

\begin{abstract}
Amodiaquine dihydrochloride monohydrate (AQ-DM) was obtained by recrystallizing amodiaquine dihydrochloride dihydrate (AQ-DD) in methanol, ethanol, and $n$-propanol. Solid-state characterization of AQ-DD and AQ-DM was performed using X-ray powder diffractometry, Fourier transform infrared spectroscopy, thermogravimetry, and differential scanning calorimetry. All recrystallized samples were identified as AQ-DM. Crystal habits of AQ-DD and AQ-DM were shown to be needle-like and rhombohedral crystals, respectively. When AQ-DD and AQ-DM were exposed to various relative humidity in dynamic vapor sorption apparatus, no solid-state interconversion was observed. However, AQ-DM showed higher solubility than AQ-DD when exposed to bulk water during solubility study, while excess AQ-DM was directly transformed back to a more stable AQ-DD structure. Heating AQ-DM sample to temperatures $\geq 190^{\circ} \mathrm{C}$ induced initial change to metastable amorphous form (AQ-DA) which was rapidly recrystallized to AQ-DD upon $\geq 80 \% \mathrm{RH}$ moisture exposure. AQ-DD was able to be recrystallized in alcohols (C1-C3) as AQ-DM solid-state structure. In summary, AQ-DM was shown to have different solubility, moisture and temperature stability, and interconversion pathways when compared to AQ-DD. Thus, when AQ-DM was selected for any pharmaceutical applications, these critical transformation and property differences should be observed and closely monitored.
\end{abstract}

KEYWORDS: amodiaquine dihydrochloride; physicochemical characterization; recrystallization; solidstate characterization; solid-state interconversion.

\section{INTRODUCTION}

Solid-state chemistry has been known to play a pivotal role in determining success or failure during the drug development life cycle $(1,2)$. Various polymorphs, amorphous, hydrates, and solvates will have an impact on the physicochemical and mechanical properties of a drug substance. During manufacturing processes, there are many factors which will affect the solid-state characteristics of a drug such as temperature, light, humidity, pressure, processing time, and solvents (3-5). These factors will not only influence the physicochemical properties of the drug, but also on their efficacy (6-9). Therefore, solid-state morphology screening of a drug is an important part in preformulation studies of solid dosage forms. The knowledge will help to understand the physicochemical properties related to their solid-state morphologies

\footnotetext{
${ }^{1}$ Faculty of Pharmaceutical Sciences, Chulalongkorn University, Bangkok, Thailand.

${ }^{2}$ Chulalongkorn University Drug and Health Products Innovation Promotion Center (CU.D.HIP), Faculty of Pharmaceutical Sciences, Chulalongkorn University, 254 Phyathai Road, Pathumwan, Bangkok, 10330, Thailand.

${ }^{3}$ To whom correspondence should be addressed. (e-mail: narueporn.s@chula.ac.th)
}

in order to prevent the transformation during manufacturing and to design properly controlled processes $(10,11)$.

Amodiaquine is one of the effective monotherapy drugs currently used as antimalarials. It is a derivative of quinolines, and it is more effective than chloroquine against resistant malarial parasites (12-15). Amodiaquine (AQ), a 4-[(7-chloro-4quinolinyl)amino]-2-[(diethyl-amino)methyl]phenol, exists as dihydrochloride salt in anhydrous, monohydrate, and dihydrate forms (16). The three dimensional crystal structures of amodiaquine were reported in the forms of its free base and tetrachlorocobaltate (II) (17). However, the above known solidstate morphologies of AQ in correlation with their physicochemical properties have not yet been reported. Therefore, the objective of this study is to evaluate the differences in amodiaquine dihydrochloride solid-state morphology after recrystallizing in various alcoholic solvents (18-21) and correlate them with their respective physicochemical properties. Methanol, ethanol, and $n$ propanol, which are monohydric alcohols, were selected as recrystallizing solvents due to their increasing series in carbon number and have not been used as pure form for AQ solidstate morphology screening in all previous reports. The result of this study may prove to be beneficial for the pharmaceutical industry in understanding the changes in physicochemical properties due to differences in AQ solid-state structures and enables the research and development scientists to select the most appropriate form for their future product development. 


\section{MATERIALS AND METHODS}

\section{Materials}

Amodiaquine dihydrochloride dihydrate (AQ-DD) reference standard was obtained from USP (Rockville, MD). AQ-DD, used as the starting material for recrystallization, was purchased from Sigma-Aldrich (St. Louis, MO). Recrystallization solvents used in solid-state morphology screening such as methanol (Burdick \& Jackson, Ulsan, Korea), anhydrous ethanol (Carlo Erba, Val de Reuil, France), and $n$-propanol (Ajax Finechem Pty Ltd., Auckland, New Zealand) were obtained from their manufacturers.

\section{Methods}

\section{Solid-State Screening and Identification}

AQ-DD starting material was recrystallized in methanol, ethanol, and $n$-propanol. When AQ-DD was initially added into the above alcoholic solvents at $30^{\circ} \mathrm{C}$, turbid liquids were observed. Mixtures were then heated to $50^{\circ} \mathrm{C}$ for additional $10 \mathrm{~min}$ until clear solutions were obtained. The solution was then cooldown in a circulating water bath (Polystat Control cc1, Huber, Germany) to a controlled temperature of $30^{\circ} \mathrm{C}$ until small crystal nuclei appeared. The sample temperature was then controlled at $30^{\circ} \mathrm{C}$ to allow crystal growth to occur and mature. The fully grown crystals were harvested and washed by each relevant alcohol-recrystallizing solvent. The crystals were then allowed to dry at controlled room temperature.

The solid-state morphology of recrystallized samples were identified by various solid-state analytical techniques: $\mathrm{X}$-ray powder diffractometry, Fourier transform infrared spectrophotometry (FT-IR), and thermogravimetry (TGA).

$X$-ray Powder Diffractometry. The starting material and the recrystallized samples were analyzed for their crystal structures by X-ray powder diffractometry (XRPD) using Miniflex II (Rigaku, Japan). Wide-angle XRPD using $\mathrm{CuK} \alpha$ radiation at $40 \mathrm{kV}$ and $20 \mathrm{~mA}$ was employed. The scan speed was held constant at $1^{\circ} 2 \theta$ per min, and the angular scanning range was programmed from 5 to $40^{\circ} 2 \theta$.

Fourier Transform Infrared Spectrophotometry. The samples were thoroughly mixed with dried $\mathrm{KBr}$ powder and finely ground in an agate mortar. The sample $\mathrm{KBr}$ mixtures were then transferred between two stainless steel punches and compressed with a hydraulic press to form compact pellets. Infrared spectra of samples were obtained by an infrared light source at 20 scans and $4.00 \mathrm{~cm}^{-1}$ resolution. The spectral wave number was collected from 4000 to $400 \mathrm{~cm}^{-1}$ by Spectrum One Fourier transform infrared spectrophotometer (Perkin Elmer, USA).

Thermogravimetric Analysis. Weight loss of samples due to increase in temperatures were determined by thermogravimetric analysis (TGA). TGA studies were carried out using TGA/SDTA851 ${ }^{\mathrm{e}}$ (Mettler Toledo, Switzerland). Accurately weighed approximately $2 \mathrm{mg}$ of the sample in $70 \mu \mathrm{l}$ alumina sample holder. The scanning rate was scheduled at $10^{\circ} \mathrm{C} / \mathrm{min}$ under a nitrogen purge gas of $60 \mathrm{ml} / \mathrm{min}$ and the scanning temperature ranged from 25 to $250^{\circ} \mathrm{C}$. Percentage weight loss was calculated and compared to the original sample weight.

\section{Physicochemical Characterization}

The starting material and the recrystallized samples were characterized for their physicochemical properties by various techniques as follow.

Scanning Electron Microscopy. The external habits were observed in detail by scanning electron microscope (SEM). Samples were carefully placed on the metal stub of SEM JSM5410LV (JEOL, Japan). They were then sputter-coated with gold under vacuum before their morphology were recorded.

Differential Scanning Calorimetry. Thermal behaviors of samples were evaluated by differential scanning calorimetry (DSC) using DSC $822^{\mathrm{e}}$ (Mettler Toledo, Switzerland). Accurately weighed approximately $3 \mathrm{mg}$ of sample in $40 \mu \mathrm{l}$ standard aluminum pan. The pan was sealed with a lid punctured with one pin hole. The scanning rate was held constant at $10^{\circ} \mathrm{C} / \mathrm{min}$, and the scanning temperature range was from 25 to $250^{\circ} \mathrm{C}$ under nitrogen purge gas of $60 \mathrm{ml} / \mathrm{min}$.

Dynamic Vapor Sorption. Transformation of the crystalline samples due to moisture was monitored by dynamic vapor sorption (DVS) apparatus (DVS Intrinsic, Surface Measurement Systems Ltd., UK). Adsorption isotherms were obtained at controlled temperature of $30^{\circ} \mathrm{C}$. The samples were exposed to an increment increase in relative humidity $(\mathrm{RH})$ from $0 \% \mathrm{RH}$ to $100 \% \mathrm{RH}$. Changes in the sample weight were periodically recorded.

Aqueous Solubility. The starting material and recrystallized samples were added to $14 \mathrm{ml}$ purified water in excess and immersed in circulating water bath (Polystat Control cc1, Huber, Germany) at a controlled temperature of $30^{\circ} \mathrm{C}$. Samples were withdrawn at intervals of 5, 10, 15, 20, 25, 30, 40, 50, 60, 90, 120, and $180 \mathrm{~min}$ to determine the amounts of drug dissolved. Aliquots were filtered through $0.45 \mu \mathrm{m}$ membrane filter and quantitatively analyzed by UV-spectrophotometry (UV-160A, Shimadzu, Japan) at $342 \mathrm{~nm}$ using $1 \times 1 \mathrm{~cm}$ quartz sample cell holder. The calibration curve was obtained by dissolving known amounts of AQ-DD in purified water and adjusted to suitable dilutions.

\section{RESULTS}

The solid-state morphology of AQ-DD after recrystallization in various alcohol solvents were characterized by appropriate solid-state analytical techniques.

\section{Solid-State Screening and Identification}

The starting material and recrystallized samples were identified for their solid-state morphology by XRPD. XRPD is considered as one of the most reliable and acceptable technique used for solid-state identification (22-24). XRPD pattern of the starting material is shown in Fig. 1, which conforms to amodiaquine dihydrochloride dihydrate (AQ-DD) reported by Llinàs et al. (16). All XRPD patterns of the recrystallized crystals from methanol (AQ $\mathrm{MeOH}$ ), ethanol (AQ $\mathrm{EtOH}$ ) and $n$-propanol (AQ $\mathrm{PrOH})$ are found to be the same, but are significantly different from the starting material, AQDD. XRPD diffractogram of AQ-DD illustrates major peaks at $6.6,8.9,10.47,12.9,19.8,20.7,25.7,26.5,28.5,33.3$, and 


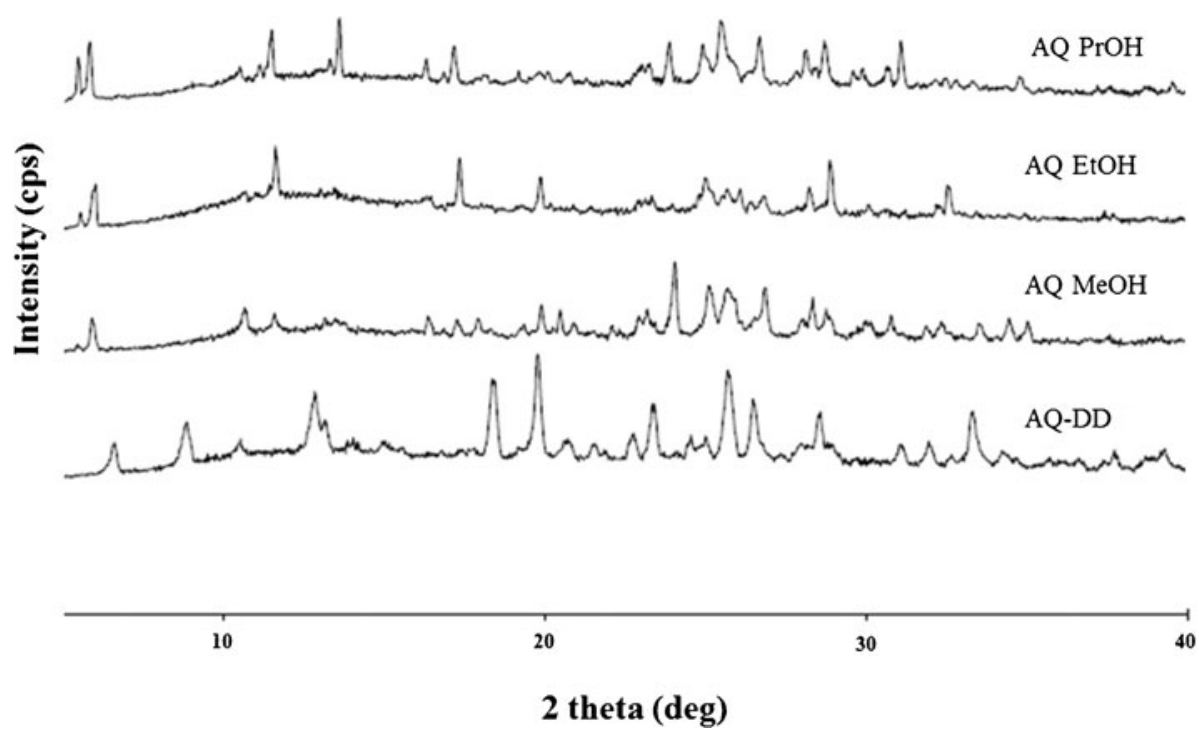

Fig. 1. XRPD diffractograms of $A Q-D D$ before and after recrystallization in methanol (AQ $\mathrm{MeOH})$, ethanol (AQ EtOH), and $n$-propanol (AQ PrOH)

$34.2^{\circ} 2 \theta$ which are absent in the diffractograms obtained from the recrystallized samples. On the other hand, peaks shown at 5.6, 6.0, 11.6, 17.3, and $26.1^{\circ} 2 \theta$ are absent in AQ-DD but clearly present in the recrystallized samples.

To identify and differentiate the intermolecular interactions, AQ-DD and recrystallized AQ-DD were characterized by FT-IR. FT-IR spectra of AQ-DD and the recrystallized samples are shown to be different, as can be seen in Fig. 2. The spectrum of AQ-DD shows prominent IR peaks at 2637 and $3406 \mathrm{~cm}^{-1}$ indicating -OH stretching and -NH stretching, respectively (25). However, the spectra of all recrystallized samples show that $-\mathrm{OH}$ stretch shifted to lower wavenumber of $2449 \mathrm{~cm}^{-1}$ and $-\mathrm{NH}$ stretch also shifted to lower wavenumber of $3233 \mathrm{~cm}^{-1}$.

To confirm the hydrate stoichiometry of AQ-DD starting material and the recrystallized samples, TGA technique was used. From preliminary TGA results, all recrystallized samples exhibit the same weight loss. Thus, AQ-EtOH is chosen as a representative for recrystallized solids and the TGA result is shown in Fig. 3. AQ-DD and AQ EtOH were heated with the same heating rate of $10^{\circ} \mathrm{C} / \mathrm{min}$. TGA thermogram of AQDD illustrates a mass loss of approximately $7 \%$ by weight after $148^{\circ} \mathrm{C}$ which was calculated to be equivalent to two moles of water. However, TGA thermogram of AQ EtOH displays only one step weight loss at higher temperature of $170^{\circ} \mathrm{C}$ with only $4 \%$ weight decrease. This weight change was calculated to be equivalent to only one mole of water.

The TGA results suggest that AQ-DD starting material is presented as dihydrate (16), while all recrystallized crystals are found to be amodiaquine dihydrochloride monohydrate and, henceforth, will be called AQ-DM.

\section{Physicochemical Characterization}

AQ-DD and AQ-DM were further evaluated for their physicochemical properties. AQ-DD crystals are visually observed to be in pale yellowish hue (Fig. 4a), while AQ-DM is presented as deep orange (Fig. 4b). Habits of both crystal forms were characterized by SEM. AQ-DD shows fine, needle-like habit, whereas AQ-DM exhibits larger rhombohedrons as shown in Fig. 4a and 4b, respectively.

Thermal properties of AQ-DD and AQ-DM were determined by DSC. AQ-DD and AQ-DM were both heated at a heating rate of $10^{\circ} \mathrm{C} / \mathrm{min}$. DSC thermogram of $\mathrm{AQ}-\mathrm{DD}$ in Fig. 5 shows a large endothermic melting peak at onset temperature of approximately $150^{\circ} \mathrm{C}$. However, DSC thermogram of AQ-DM shows one major and two minor endothermic events at onset temperatures of approximately 170, 190, and $215^{\circ} \mathrm{C}$, respectively.

From the results obtained by TGA on AQ-DM (Fig. 3), a steady weight was achieved from 190 to $250^{\circ} \mathrm{C}$. However, when evaluated by DSC, there are two additional thermal events occurring at approximately 190 and $215^{\circ} \mathrm{C}$. These questionable thermal events were further evaluated by heating AQ-DD and AQ-DM to 190,215 and $250^{\circ} \mathrm{C}$ and then paused experiments to collect samples for further evaluation by XRPD. AQ-DM diffractograms in Fig. 6b show that at $190^{\circ} \mathrm{C}$ residual AQ-DM crystalline pattern is still observable while at 215 and $250^{\circ} \mathrm{C}$ two amorphous halo pattern are seen. Previous TGA results show no observable weight change occurring at 190 to $250^{\circ} \mathrm{C}$. It can be explained that the first large endothermic event is a loss of one water molecule while resulting in a crystalline anhydrous structure but retaining the monohydrate XRPD pattern. The following two smaller endotherms are due to the modification of this anhydrous structure to a higher energetically preferred amorphous state at $215^{\circ} \mathrm{C}$ and eventually degrade at $250^{\circ} \mathrm{C}$. However, AQ-DD diffraction patterns after heating and stopping to collect samples at 190,215 , and $250^{\circ} \mathrm{C}$ all show halo-amorphous structure (Fig. 6a). These amorphous species will be called AQ-DA.

The sensitivity of crystals to water vapor was determined by dynamic vapor sorption (DVS) analysis. Relative humidity in the environment may vary according to the differences in geographical locations and seasons, which, in turn, could affect the stability of solid-state forms of drugs $(26,27)$. Therefore, the ability of water to adsorb on the surface of each solid-state structure was evaluated by isothermal DVS at $30^{\circ} \mathrm{C}$ within controlled relative humidity range of $0-100 \% \mathrm{RH}$. The results 


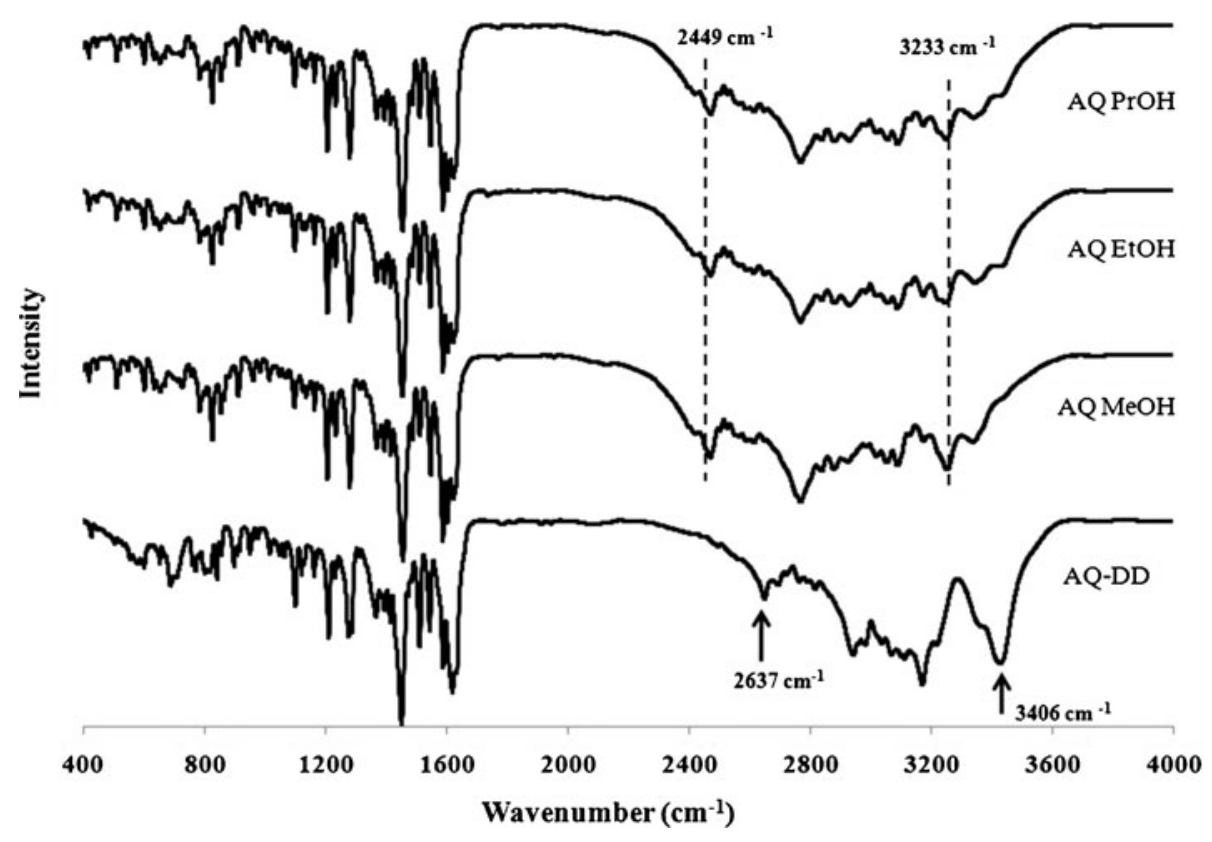

Fig. 2. FT-IR spectra of AQ-DD before and after recrystallization in methanol (AQ MeOH), ethanol (AQ EtOH), and $n$-propanol (AQ PrOH)

show that only negligible amount of water is adsorbed on surfaces of both AQ-DD and AQ-DM with total amount of moisture adsorbed (equilibrated at $100 \% \mathrm{RH}$ ) of only 1.44 and $0.44 \%$ by weight, respectively (Fig. 7 ).

However, AQ-DA, obtained by removal of water from AQ-DD and AQ-DM, is found to have different behavior. AQ-DA abruptly adsorbed water vapor to $18 \% \mathrm{w} / \mathrm{w}$ of its original weight at $80 \% \mathrm{RH}$. After $80 \% \mathrm{RH}$, the structure releases significant amount of moisture down to approximately $7-8 \%$. No further change in weight is observed during desorption cycle from $100 \% \mathrm{RH}$ to $0 \% \mathrm{RH}$. The sample weight remains constant at approximately $7-8 \% w / w$ throughout the rest of the experiment.

Equilibrium solubility in water for both crystal forms, AQ-DD and AQ-DM, were conducted at $30^{\circ} \mathrm{C}$. AQ-DD is increasingly soluble until $30 \mathrm{~min}$ where it reached equilibrium at $47.70 \mathrm{mg} / \mathrm{ml}$ (Fig. 8). On the other hand, solubility behavior of AQ-DM is dramatically different from AQ-DD. During the first $15 \mathrm{~min}$, AQ-DM shows solubility as high as $95 \mathrm{mg} / \mathrm{ml}$. Twofolds higher than the solubility of AQ-DD during the

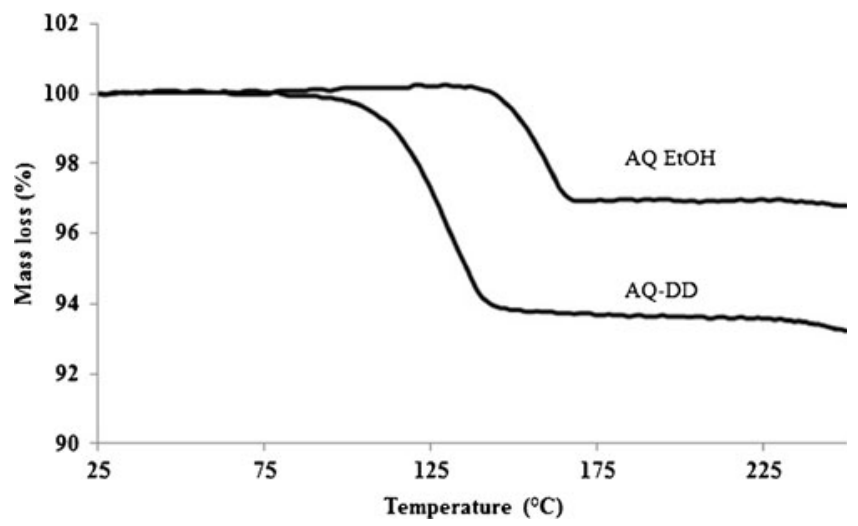

Fig. 3. TGA thermograms of AQ-DD before and after recrystallization in ethanol (AQ EtOH) same time period. After $15 \mathrm{~min}$, solubility of AQ-DM greatly decreased and reached the same final saturated solubility of AQ-DD at $46.5 \mathrm{mg} / \mathrm{ml}$ from $120 \mathrm{~min}$ onward. The color of dispersed solids in the AQ-DM solubility vessel also change from bright orange during the first 15 min to pale yellow at the end of the experiment. No change in color is observed during AQ-DD solubility study where pale yellow solution is seen throughout the experiment.

\section{DISCUSSION}

\section{Solid-State Screening and Characterization}

Amodiaquine dihydrochloride was shown to have many solid-state structures $(16,17)$. The present study focused on the evaluation of AQ-DD solid-state morphology after recrystallization in $\mathrm{C} 1-\mathrm{C} 3$ monohydric alcohols and also on the conversions of these recrystallized forms in correlation with their relevant physicochemical properties. Recrystallized crystals obtained from each alcoholic solvent were evaluated in comparison to the AQ-DD starting material by XRPD and FT-IR. XRPD diffractogram of the starting material (Fig. 1) complied with dihydrate form of amodiaquine dihydrochloride reported by Llinàs et al. (16). Whereas XRPD patterns of all recrystallized solids from alcohol were the same but significantly different from the pattern of AQDD starting material. Similarly, FT-IR spectra of the starting material and the three recrystallized solids were also shown to be different (Fig. 2). In addition, AQ-DD and the three recrystallized samples showed different crystal habits and color when visually observed (Fig. 4).

From these results, it can be concluded that recrystallization of AQ-DD in aliphatic alcohols with carbon series from $\mathrm{C} 1$ to $\mathrm{C} 3$ affects the final solid-state morphology of the original AQ-DD. The mechanism of modification can be best explained by solvent-solute interactions $(20,28)$. Hydrogen bond formation, between solvent and solute, plays a key role 


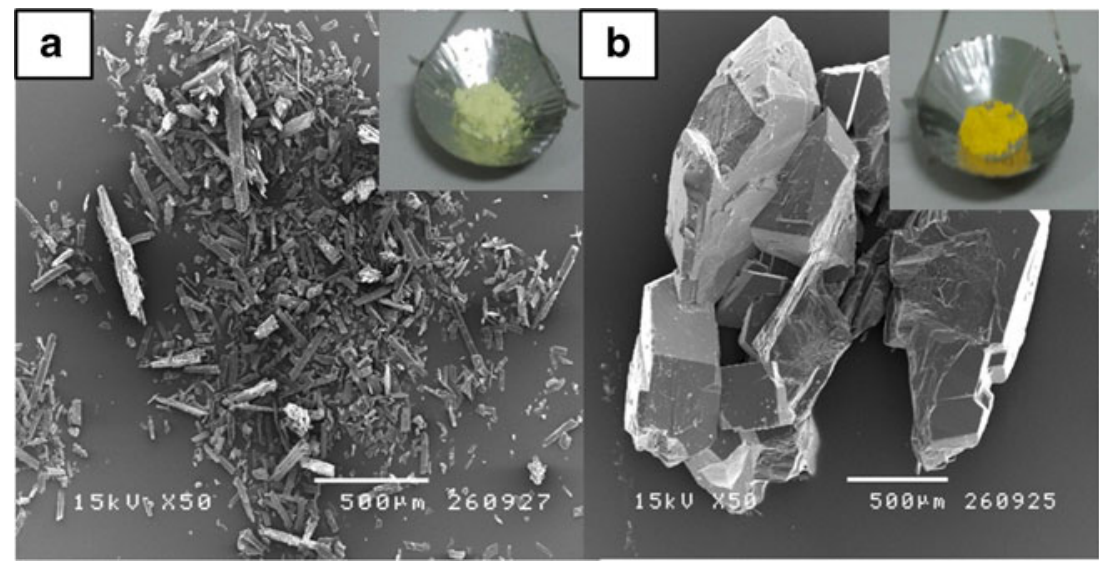

Fig. 4. Colors and habits of $\mathbf{a} \mathrm{AQ}-\mathrm{DD}$ and $\mathbf{b} \mathrm{AQ}-\mathrm{DM}$ evaluated by visual observation and SEM $(\times 50)$

in the formation of different solid-state morphology $(19,20$, 28). Normally, there are three types of solvents used in routine morphology screening. First, nonpolar aprotic solvents, such as hexane, do not interact with the solute. Second, dipolar aprotic solvents which are polar but not hydrogen bond donor, such as acetonitrile. Finally, dipolar protic solvents which are polar with hydrogen bond donor, such as water, methanol, and ethanol (20). In this study, only dipolar protic solvents were chosen because aliphatic alcohols are commonly encountered in many pharmaceutical manufacturing processes. These solvents exhibit hydrogen bond donor functional group that can interact with amodiaquine dihydrochloride via hydrogen bond formation, which is different than water, and caused morphology rearrangement of original AQ$\mathrm{DD}$ to the resulting recrystallized form (20). Almandoz and coworkers (21) addressed in their study that the hydrogen bond donor capacity $(\alpha)$ of methanol, ethanol, and $n$-propanol are $0.98,0.86$, and 0.84 , respectively. The report confirms that the polarities between these alcoholic recrystallizing solvents are only slightly different and not sufficient to initiate different individual crystalline forms of amodiaquine dihydrochloride in our study. Consequently, the recrystallized crystals of amodiaquine dihydrochloride obtained from these three aliphatic alcohols showed the same solid state morphology but very different from the original AQ-DD raw material.

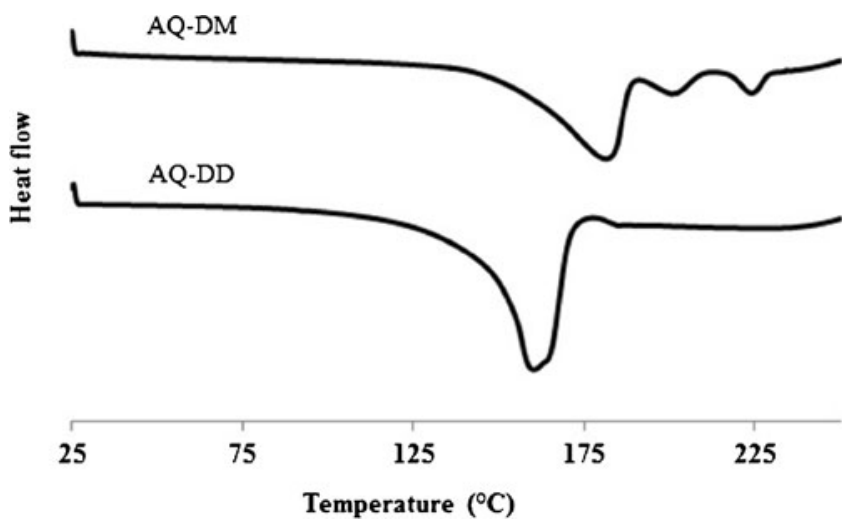

Fig. 5. DSC thermograms of AQ-DD and AQ-DM at a heating rate of $10^{\circ} \mathrm{C} / \mathrm{min}$ from 25 to $250^{\circ} \mathrm{C}$
Thermal analyses using DSC and TGA were performed to confirm the differences in solid-state morphology of recrystallized solids. DSC thermogram of the starting material (Fig. 5) displays only one endothermic dehydration peak at approximately $148^{\circ} \mathrm{C}$ similar to TGA thermogram (Fig. 3) which shows approximate weight loss of $7 \% \mathrm{w} / \mathrm{w}$ at $150^{\circ} \mathrm{C}$. This endothermic event occurring at $148^{\circ} \mathrm{C}$ was calculated and found to be due to the loss of two moles of water, confirming that the starting material was amodiaquine dihydrochloride "dihydrate" (AQ-DD). In the case of the recrystallized solids, DSC thermograms (Fig. 5) illustrate large endothermic dehydration peak at $152^{\circ} \mathrm{C}$ subsequently followed by two smaller endotherms. TGA thermogram (Fig. 3) shows only one step weight loss of $4 \% w / w$ at approximately $152^{\circ} \mathrm{C}$. From this result, it can be explained that the large endothermic event was the loss of one mole of water, while the two smaller endotherms were due to solid-state structural modifications of crystalline anhydrous structure with no further weight loss observed between 190 to $250^{\circ} \mathrm{C}$. Therefore, these recrystallized crystals were amodiaquine dihydrochloride "monohydrate" (AQ-DM). The questionable thermal events of AQ-DM were further evaluated by heating AQ-DM and AQ-DD to the fixed temperatures of 190,215 , and $250^{\circ} \mathrm{C}$. The products were collected and further evaluated by XRPD (Fig. 6). This results show that when water molecules were removed from the structure of AQ-DM by heat, resulting anhydrous crystalline AQ-DM solid structure exhibited pores which were once occupied by water molecules (Fig. 9b). AQDM was found to arrange in II-II stacking orientation between phenol in one molecule and quinolone ring in another $(17,29)$. After dehydrating AQ-DM, the original structure could be retained but in an anhydrous state mainly due to the strength of II-II stacking orientation (Fig. 9b). Hence, the presence of water had less influence on the stabilization of AQ-DM anhydrous solid structure when compared to the interaction via II-II stacking. The AQ-DM anhydrous solid structure remained stable until additional heat was introduced into the system when the structure collapsed resulting in an amorphous structure as depicted in Fig. 9b. Increasing heat from this point onward will only result in degradation, hence shown by the third endotherm.

On the other hand, AQ-DD, shows different path in transformation after heat was introduced. When two moles of water were removed from AQ-DD, crystalline structure 


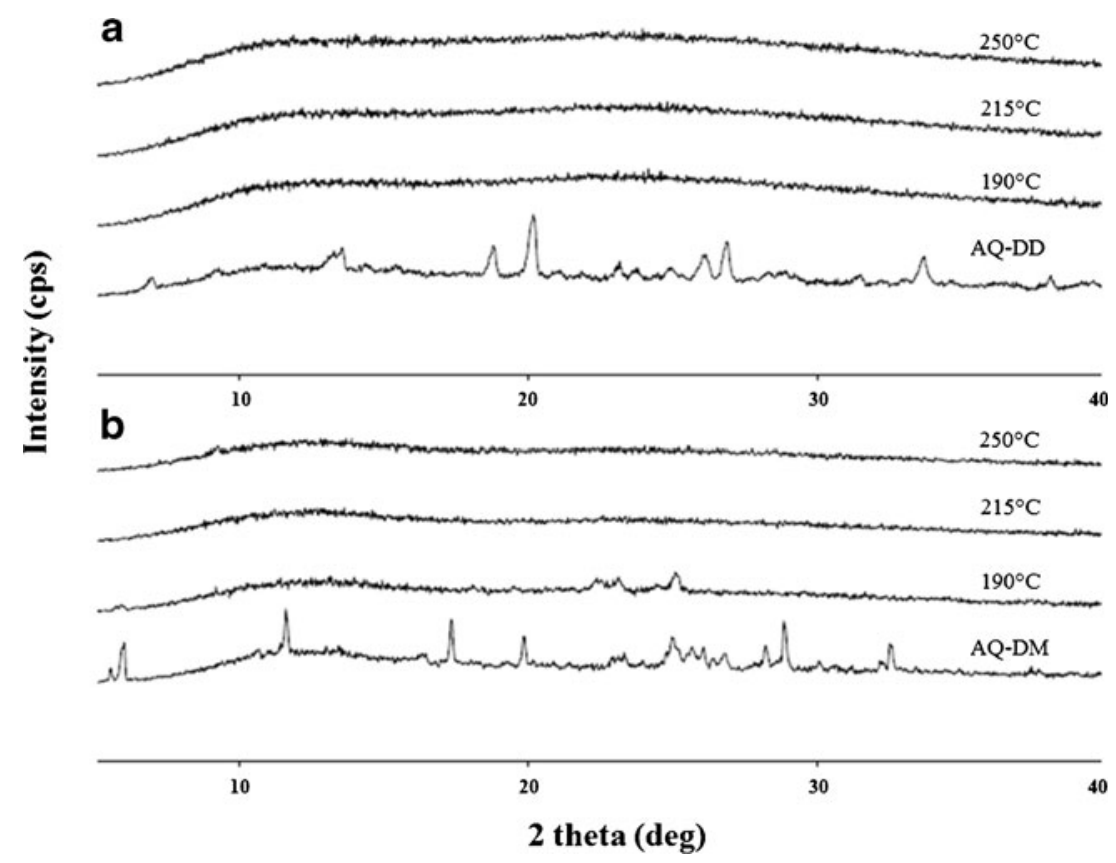

Fig. 6. XRPD diffractograms of products of a AQ-DD and b AQ-DM collected from heating by DSC (heating rate $10^{\circ} \mathrm{C} / \mathrm{min}$ ) to the designated temperatures of 190,215 , and $250^{\circ} \mathrm{C}$

collapsed immediately, resulting in a randomly oriented arrangement. This sudden collapse in crystalline AQ-DD after water removal was due to the initiation of highly porous solid which these pores were once occupied by water with very weak lattice strength and could not withhold the original crystalline structure. This finding was in accordance with studies by Llinàs et al. and Semeniuk et al. $(16,17)$, where water molecules in AQ-DD solid structure played crucial role in maintaining the dihydrate crystal packing. Water was shown to function as hydrogen bond bridges holding drug molecules together in its dihydrate solid-state structure. If by any circumstances water was removed, the structure will collapse because of the instability of the crystal lattice (Fig. 9a).

\section{Physicochemical Characterization}

AQ-DD and AQ-DM were evaluated for their stability under stressed conditions with water in the states of vapor and liquid. AQ-DD and AQ-DM adsorbed only negligible amount of water vapor on their surfaces under isothermal dynamic

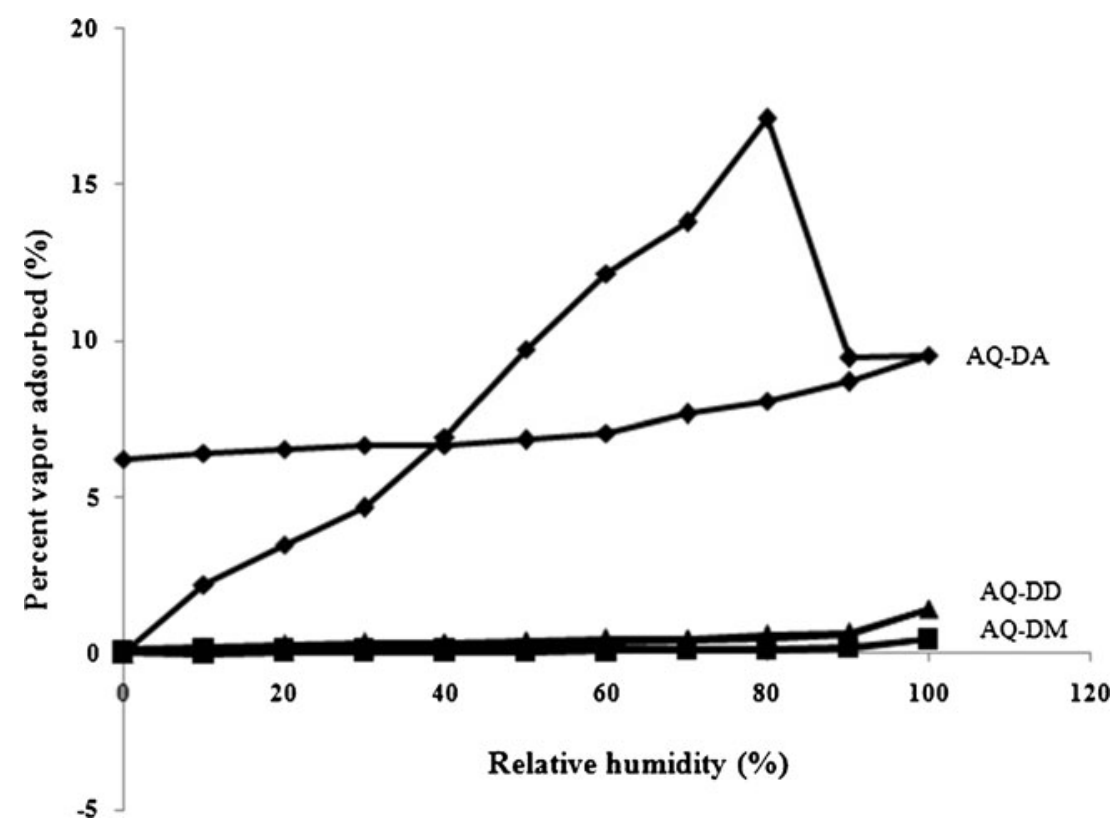

Fig. 7. Amount of water vapor on surfaces of AQ-DD, AQ-DM, and AQ-DA crystals at $30^{\circ} \mathrm{C}$ during moisture adsorption-desorption cycles using DVS 


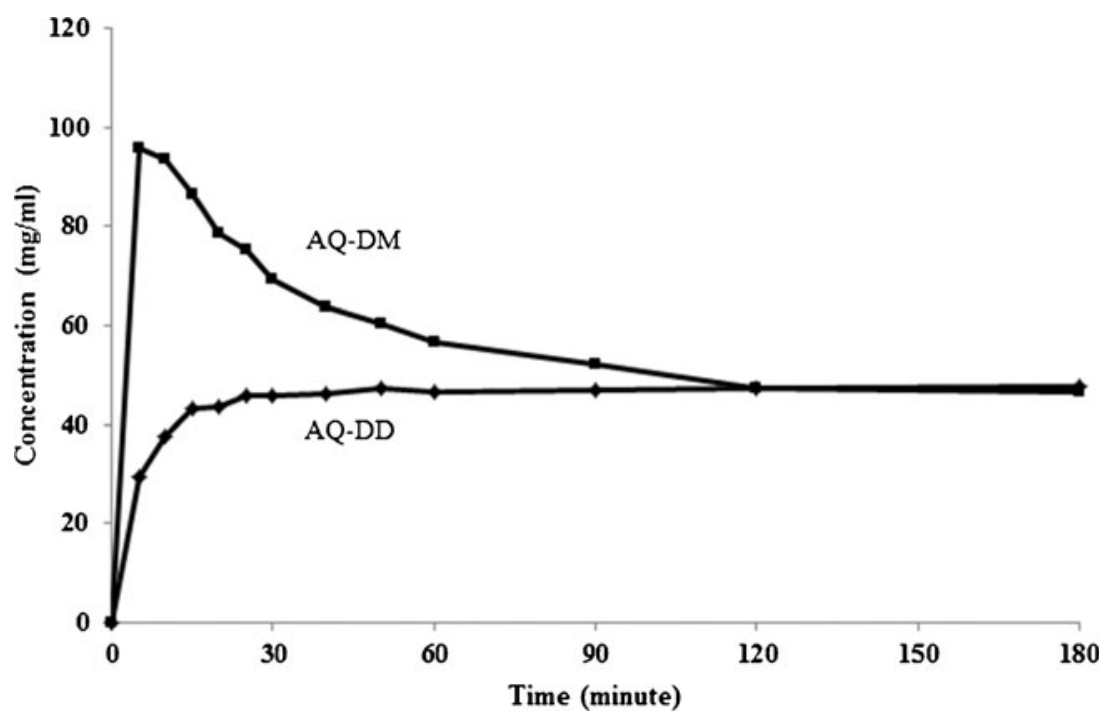

Fig. 8. Equilibrium water solubility profiles of AQ-DD and AQ-DM at $30^{\circ} \mathrm{C}$

vapor sorption condition from $0 \% \mathrm{RH}$ to $100 \% \mathrm{RH}$. It could be concluded that both solid-state forms are nonhygroscopic and will not uptake moisture in the form of vapor or gas (30). In addition, AQ-DD adsorbed slightly higher amount of water than AQ-DM owing to smaller crystal size (Fig.4a, b), leading to slightly higher surface area. However, no solid-state transformation occurred between the two forms after exposure to water vapor. However, when AQ-DA was exposed to the same dynamic water vapor condition, it gradually takes up moisture to approximately $18 \% w / w$ at $80 \% \mathrm{RH}$. At this time, molecular mobility of the drug increased to a point where preferred recrystallization to lower energetic crystalline phase occurred due to high water content within the solid structure. Moisture was released down to approximately $7 \% \mathrm{w} / \mathrm{w}$ between $80 \%$ RH to $100 \%$ RH. Desorption cycle from $100 \%$ RH to $0 \% \mathrm{RH}$ did not induce further weight loss. Final weight remained constant at $7 \% \mathrm{w} / \mathrm{w}$ even at $0 \% \mathrm{RH}$ indicating the recrystallization to AQ-DD.
Aqueous solubility of both crystal forms was evaluated at $30^{\circ} \mathrm{C}$. Water molecule is considered as dipolar protic solvent with a strong hydrogen bond donor capacity $(\alpha)$ equals to 1.17 (21). In the first $15 \mathrm{~min}$, solubility of AQ-DM was significantly higher than AQ-DD. Solubility of both forms reached the same equilibrium plateau concentrations at $120 \mathrm{~min}$. The possible explanation for this occurrence could be that when both forms were exposed to bulk water, excess AQ-DD solid structure was already saturated with hydrogen bonds as reported by Llinàs et al. (16). Additional hydrogen donor supplied by bulk water would not interfere with the stable AQ-DD solid arrangement, resulting in the true solubility value of AQ-DD with no interconversion during the study. However, the monohydrate structure was reported to be deficient in hydrogen bond donors as $\mathrm{Cl}^{-}$ions formed only one hydrogen bond instead of two or three as reported by Llinàs et al. (16). Thus, water molecules in the medium possibly act as instant hydrogen bond donor to the monohydrate structure, dissolving the drug resulting in initially

a

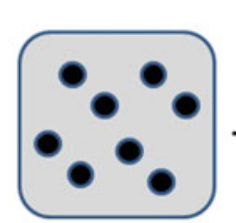

AQ-DD

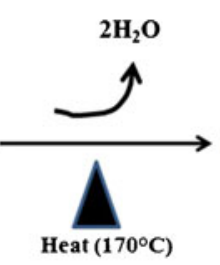

Heat $\left(170^{\circ} \mathrm{C}\right)$

Peak 1

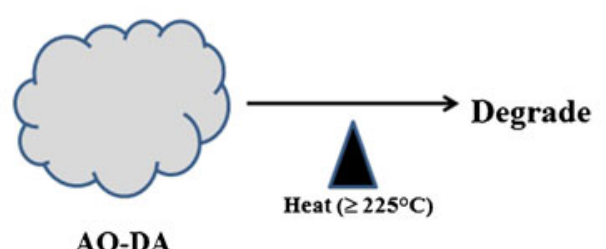

AQ-DA
Heat $\left(\geq 225^{\circ} \mathrm{C}\right)$ b

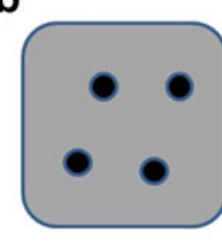

AQ-DM

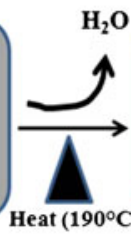

Peak 1

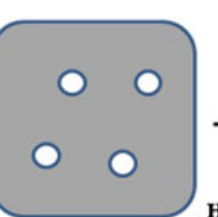

AQ-DM anhydrous solid structure

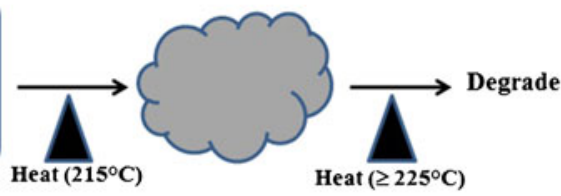

AQ-DA

Peak 3

Fig. 9. Schematic presentations of solid structural conversions of AQ-DD and AQ-DM after dehydration by DSC (heating rate $10^{\circ} \mathrm{C} / \mathrm{min}$ from 25 to $250^{\circ} \mathrm{C}$ ) 
high solubility value. Eventually, molecular rearrangement occurred to a more stable structure with optimal hydrogen bond scheme, AQ-DD. AQ-DM would initially show higher solubility and eventually recrystallized out as a more stable hydrogen bond-rich AQ-DD. From the vapor sorption and solubility results, it could be summarized that water vapor in the surrounding environment was not sufficient to induce crystal structure transformation of both AQ-DD and AQ-DM. It may be due to the fact that both crystal forms are nonhygroscopic; therefore, water molecules in gaseous state could not adsorbed on the surfaces to a sufficient extent to induce phase transformation within the crystal lattice (30). AQ-DA, however, would convert to AQ-DD at sufficiently high moisture environment $(80 \% \mathrm{RH})$ due to its natural hygroscopic amorphous behavior. Also, during solubility evaluation, AQ-DM phase transformation occurred to a more stable form, AQ-DD. Thus, the color of the dispersion was changed from bright orange due to excess AQ-DM to pale yellow of AQ-DD during the study. This was due to the intimate exposure to bulk water in liquid state and the hydrogen bond donor capacity of water. When AQ-DM was in contact with liquid water, molecules of the drug initially solubilized out until supersaturation was reached and finally recrystallized out as a more stable AQ-DD with reduced solubility (Fig. 10) $(28,31)$.

\section{CONCLUSION}

AQ-DD recrystallized in aliphatic alcohols (methanol, ethanol, and $n$-propanol) are all shown to form AQ-DM. Thermal properties and XRPD diffractograms of AQ-DD and AQ-DM are different but with similar isothermic water vapor sorption behavior. The direct solid-state transformation of AQ-DM to AQ-DD only occurs in bulk liquid water and not by exposing to water vapor. However, AQ-DM may first indirectly converts to a metastable amorphous form (AQ-DA) by heat $\left(\geq 190^{\circ} \mathrm{C}\right)$ before eventually transforming to AQ-DD by final exposure to water vapor of $\geq 80 \% \mathrm{RH}$. The transformation pathways obtained from this study are summarized in Fig. 10. The knowledge obtained from this report will be beneficial in preventing unnecessary solid-state transformation which may occur during product development process

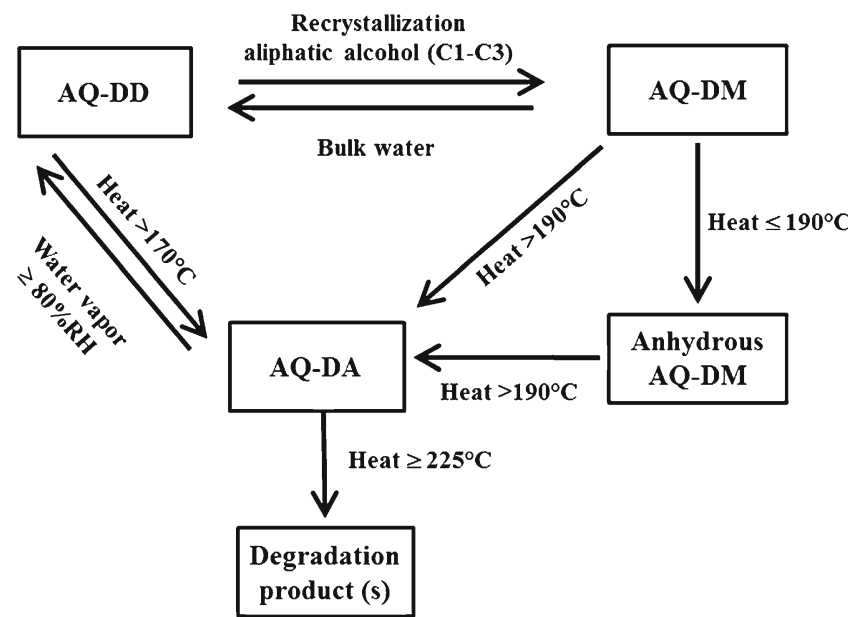

Fig. 10. Interconversion pathways of $A Q-D D$ and $A Q-D M$ due to heat and moisture and eventually alter the quality and in vivo efficacy of the drug due to physicochemical property variations.

\section{ACKNOWLEDGMENTS}

The authors would like to acknowledge the Chulalongkorn University Centenary Academic Development Project and Pharmaceutical Research Instrument Center, Faculty of Pharmaceutical Sciences, Chulalongkorn University for solid state analytical instrument support.

\section{REFERENCES}

1. Morris KR, Griesser UJ, Eckhardt CJ, Stowell JG. Theoretical approaches to physical transformations of active pharmaceutical ingredients during manufacturing process. Adv Drug Deliv Rev. 2001;48:91-114.

2. Tung H-H. Industrial perspectives of pharmaceutical crystallization. Org Process Res Dev. 2013;7:445-54.

3. Colleir JW, Shah RB, Gupta A, Sayeed V, Habib MJ, Khan MA. Influence of formulation and processing factor on stability of levothyroxine sodium pentahydrate. AAPS PharmSciTech. 2010;11:818-25.

4. Zong Z, Desai SD, Kaushal AM, Barich DH, Huang HS, Muanson, et al. The stabilizing effect of moisture on the solidstate degradation of gabapentin. AAPS PharmSciTech. 2011;12:924-31.

5. Airaksinen S, Karjalainen M, Kivikero N, Westermarck S, Shevchenko A, Rantanen J, et al. Excipient selection can significantly affect solid-state phase transformation in formulation during wet granulation. AAPS PharmSciTech. 2005;6:E311-22.

6. HÖrter D, Dressman JB. Influence of physicochemical properties on dissolution of drugs in the gastrointestinal tract. Adv Drug Deliv Rev. 2001;46:75-87.

7. Chieng N, Rades T, Aaltonen J. An overview of recent studies on the analysis of pharmaceutical polymorphs. J Pharm Biomed Anal. 2011:55:618-44.

8. McNamara DP, Childs SL, Giordano J, Iarriccio A, Cassidy J, Shet MS, et al. Use of a glutaric acid cocrystal to improve oral bioavailability of a low solubility API. Pharm Res. 2006;23:1888-97.

9. Blagden N, Matas M, Gavan PT, York P. Crystal engineering of pharmaceutical active ingredients to improve solubility and dissolution rates. Adv Drug Deliv Rev. 2007;59:617-30.

10. Vippagunta SR, Brittain HG, Grant DJW. Crystalline solids. Adv Drug Deliv Rev. 2001;48:3-36.

11. Byrn SR, Pfeiffer RR, Stephenson G, Grant DJW, Gleason WB. Solid-state pharmaceutical chemistry. Chem Mater. 1994;6:1148-58.

12. Ashley E, McGready R, Proulx S, Nosten F. Malaria. Travel Med Infect Dis. 2006;4:159-73.

13. Adjei GO, Kudzi W, Dodoo A, Kurtzhals JAL. Artesunate plus amodiaquine combination therapy: reviewing the evidence. Drug Dev Res. 2010;71:33-43.

14. Hawley SR, Bray PG, Park BK, Ward SA. Amodiaquine accumulation in Plasmodium falciparum as a possible explanation for its superior antimalarial activity over chloroquine. Mol Biochem Parasitol. 1996;80:15-25.

15. Davis CB, Bambal R, Moorthy GS, Hugger E, Xiang H, Park BK, et al. Comparative preclinical drug metabolism and pharmacokinetic evaluation of novel 4-amoquinoline anti-malarial. J Pharm Sci. 2009;98:362-77.

16. Llinàs A, Fábián L, Burley JC, Streek J, Goodman JM. Amodiaquinium dichloride dihydrate from laboratory powder diffraction data. Acta Crystallogr Sect E. 2006;62:4196-9.

17. Semeniuk A, Niedospial A, Kalinowska-Tluscik J, Nitek W, Oleksyn BJ. Molecular geometry of antimalarial amodiaquine in different crystalline environments. J Mol Struct. 2008;875:32-41. 
18. Allesø M, Van Den Berg F, Cornett C, Jørgensen FS, HallingSørensen B, de Diego Lopez H, et al. Solvent diversity in polymorph screening. J Pharm Sci. 2008;97:2145-59.

19. Gu C-H, Young JRV, Grant DJW. Polymorph screening: influence of solvents on the rate of solvent-mediated polymorphic transformation. J Pharm Sci. 2001;90:1878-90.

20. Mirmehrabi M, Rohani S. An approach to solvent screening for crystallization of polymorphic pharmaceutical and fine chemical. J Pharm Sci. 2005;94:1560-76.

21. Almandoz MC, Sancho MI, Blanco SE. Spectroscopic and DFT study of solvent effects on the electronic absorption spectra of sulfamethoxazole in neat and binary solvent mixtures. Spectrochim Acta A. 2014;118:112-9.

22. Gilmore CJ. X-ray diffraction. In: Storey RA, Ymén I, editors. Solid state characterization of pharmaceuticals. Chichester: Blackwell Publishing; 2011. p. 35-70.

23. Rodriguez-Spong B, Price CP, Jayasankar A, Matzger AJ, Rodriguez-Hornedo N. General principles of pharmaceutical solid polymorphism: a supramolecular perspective. Adv Drug Deliv Rev. 2004;56:241-74.

24. Yu L, Reutzel SM, Stephenson GA. Physical characterization of polymorphic drugs: an integrated characterization strategy. PSTT. 1998;1:118-27.
25. Wade Jr LG. Organic chemistry. 3rd ed. New Jersey: PrenticeHall, Inc; 1997.

26. Carvajal M, Staniforth JN. Interactions of water with the surface of crystal polymorphs. Int J Pharm. 2006;307:216-24.

27. Jayasankar A, Good DJ, Rodriguez-Hornedo N. Mechanism by which moisture generates cocrystals. Mol Pharm. 2007:4:360-72.

28. Aaltonen J, Heinänen P, Peltonen L, Kortjärvi H, Tanninen VP, Christiansen L, et al. In situ measurement of solvent-mediated phase transformations during dissolution test. J Pharm Sci. 2006;95:2730-7.

29. Yennawar HP, Viswamitra MA. Steric and rotational constaints in the X-ray structure of the antimalarial drug amodiaquine. Curr Sci. 1991;61:39-43.

30. Murikipudi V, Gupta P, Sihorkar V. Efficient throughput method for hygroscopicity classification of active and inactive pharmaceutical ingredients by water vapor sorption analysis. Pharm Dev Technol. 2013;18:348-58.

31. Variankaval N, Lee C, Xu J, Calabria R, Tsou N, Ball R. Water activity-mediated control of crystalline phases of an active pharmaceutical ingredient. Org Process Res Dev. 2007;11:229-36. 